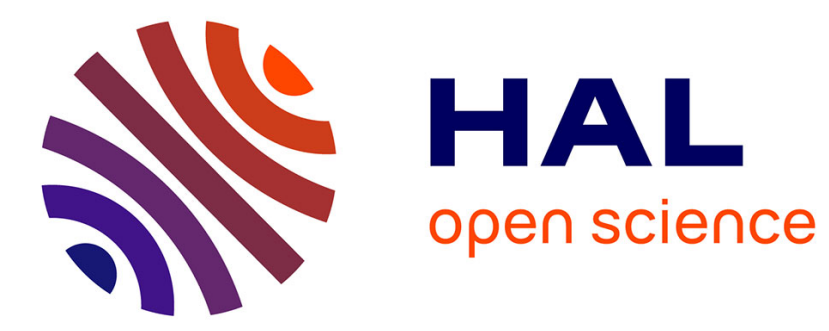

\title{
On-plate autonomous exploration for an inspection robot using ultrasonic guided waves
}

Ayoub Ridani, Othmane-Latif Ouabi, Nico F Declercq, Cédric Pradalier

\section{To cite this version:}

Ayoub Ridani, Othmane-Latif Ouabi, Nico F Declercq, Cédric Pradalier. On-plate autonomous exploration for an inspection robot using ultrasonic guided waves. European Conference on Mobile Robots, Aug 2021, Bonn, Germany. hal-03320514

\section{HAL Id: hal-03320514 https://hal.science/hal-03320514}

Submitted on 16 Aug 2021

HAL is a multi-disciplinary open access archive for the deposit and dissemination of scientific research documents, whether they are published or not. The documents may come from teaching and research institutions in France or abroad, or from public or private research centers.
L'archive ouverte pluridisciplinaire HAL, est destinée au dépôt et à la diffusion de documents scientifiques de niveau recherche, publiés ou non, émanant des établissements d'enseignement et de recherche français ou étrangers, des laboratoires publics ou privés. 


\title{
On-plate autonomous exploration for an inspection robot using ultrasonic guided waves
}

\author{
Ayoub Ridani ${ }^{1}$, Othmane-Latif Ouabi ${ }^{1}$, Nico F. Declercq ${ }^{1,2}$ and Cédric Pradalier ${ }^{1}$
}

\begin{abstract}
This article presents an active-sensing strategy based on frontier exploration to enable the autonomous reconstruction of the geometry of a metal surface by a mobile robot relying on ultrasonic echoes. Such a strategy can be beneficial to the development of a fully autonomous robotic agent for the inspection of large metal structures such as storage tanks or ship hulls. The developed method relies on a grid map generated by detecting the first echo within the measurements referring to the closest edge to the sensor, and it employs a utility function that we define to balance travel cost and information gain using an estimation of the plate geometry obtained via beamforming. Next, the sensor is directed to the next best location. The developed method is evaluated in simulation and compared with multiple algorithms, essentially closest and random frontier point selection. Finally, an experiment using a mobile robot equipped with co-localized pair of transducers is used to validate the viability of the approach.
\end{abstract}

\section{INTRODUCTION}

Monitoring the health of structures is a major stake (if not critical) in many different fields of Industry. Ultrasoundbased inspection methods have been identified as a promising solution for the long-range inspection of materials. Structures under consideration are usually made of metal (e.g., pipes, rails...) or composite materials (e.g., plane outer shapes...).

On the one hand, most of the recent ultrasound-based inspection methods are meant to be deployed on static networks of sensors permanently attached to the structure, with an application to Structural Health Monitoring (SHM). However, such methods can only be used to monitor a very restricted area. On the other hand, ultrasound-based autonomous robotic inspection has not yet been established, whereas it could benefit from the agent mobility to precisely assess, through acoustic tomography, the integrity of large structures such as ship hulls or storage tanks.

While precise localization of a mobile agent on a platebased metal structure has been identified as a fundamental requirement to long-range robotic inspection [1], Ultrasonic Guided Waves (UGWs) have proven to be useful for both plate geometry reconstruction and on-plate robot localization, yielding an innovative solution to the SLAM problem [2]. Yet, this solution has been assessed without using a real robotic platform and with pre-defined paths.

*This work is part of the BugWright2 project. This project is supported by the European Commission under grant agreement 871260 - BugWright2.

${ }^{1}$ Ayoub Ridani, Othmane-Latif Ouabi, Nico F. Declercq and Cédric Pradalier are with the International Research Lab Georgia Tech - CNRS, IRL 2958, Metz, France. \{aridani, oouabi, cedric.pradalier\}egeorgiatech-metz. fr

${ }^{2}$ Nico F. Declercq is also with Georgia Institute of Technology, Atlanta, GA 30332-0250, USA. declercqegatech. edu

978-1-6654-1213-1/21/\$31.00 @2021 IEEE

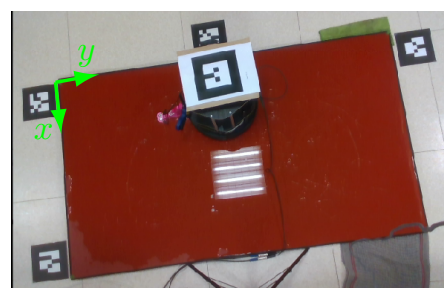

(a)

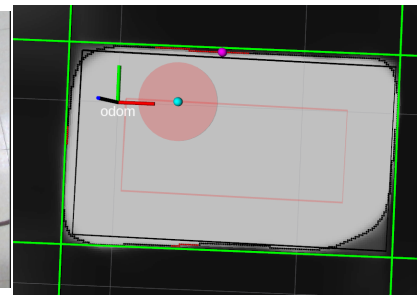

(b)
Fig. 1: Representative intermediate result for the proposed method in a realworld scenario. (a) Computed In-plate Points Grid. The blue dot refers to the sensor position whereas the purple dot is the goal position. The green lines represent the estimated plate edges and valid (resp. invalid) frontier points are the red (resp. black) dots. (b) Representation of the associated configuration on the lab experiment.

From a robotic perspective, plate geometry reconstruction and defect detection can be considered as a mapping problem, for which autonomous solutions are desirable [1]. Autonomous robotic exploration is a major research issue in robotics incorporating the aspect of how to make decisions for the next actions to maximize information gain and minimize costs. Frontier-based approaches yield common solutions to the exploration problem and are extensively used to map indoor environments. For our application, such strategies may be leveraged to determine relevant robot trajectories, to achieve fast and accurate reconstruction of the plate geometry, and under the constraint that the robot stays on the plate during the reconstruction, without crossing any boundary.

In this work, we consider a mobile unit equipped with a pair of co-localized piezoelectric transducers for emission and reception of ultrasonic waves as presented in Fig. 1b. In contrast with standard sensing technology (range-finder laser, ultra-wideband beacons, sonar...), the exploitation of guided waves measurements in a pulse-echo setup is more challenging due to their dispersive nature which causes wavepacket deformation when the propagation distance is large, but also due to the overlapping of the multiple reflections of the incident wave on the plate boundaries, which results in diffuse mixture data.

In [2], a map building technique is presented in which we estimate the boundaries of a rectangular plate. Here, we aim at pursuing this work by introducing an autonomous exploration technique integrating UGWs and exploiting the estimated plate geometry while limiting ourselves to flat rectangular plates. Frontier exploration is taken as the basic framework for exploration and we use a grid map, that we call In-plate Points Grid illustrated in Fig. 1a, computed using the detection of the closest edge to the sensor and 
the plate estimation to define areas that are inside the plate such that during robot motion, the sensor remains on the plate preventing the plate's geometry from being lost. The contributions of this paper are summarized as follows:

1) A demonstration of the utility of using the In-plate Points Grid map in combination with the geometry estimator [2] to map a "safe space" that ensures the sensor remains on the plate during robot motion.

2) An integration of UGWs sensing modality with the frontier exploration approach that balances information gain and travel cost.

3) A demonstration of the method's validity on simulation and real-world scenarios using a mobile robot on a rectangular metallic plate.

\section{RELATED WORK}

Robotics exploration, which uses mobile robots to map unknown environments, has been studied for years. Among the various proposed methods, frontier-based exploration is one of the classical approaches [3]. The key to effective frontier exploration is the selection of target frontier points. In the original method [3], the closest frontier is selected as the next target. In most cases, exploration strategies select the next best frontier by evaluating candidate locations according to different criteria resulting in various extensions of the basic frontier-based exploration strategy [4]-[6]. In [7] a utility function balancing the travel cost with the information gain is defined. In [8], a more principled approach to aggregate criteria, based on multi-objective optimization, is proposed. Currently, two methods are applied to compute information gain: one uses direct measurements of undetected space size in the visible region of the target frontier point [8], and the other uses the information entropy method [9]. In both cases, the nominal sensor range is used to define the region expected to be explored for a given frontier point. In our case, using the sensor range to compute information gain is inappropriate because Lamb waves propagate over long distances in metallic plates exceeding the standard dimensions of these plates.

On the other hand, [10] recently introduced Lamb wavebased frontier exploration strategy (LFE), demonstrating the potential contribution of Lamb wave-based sensing to the field of mobile robot exploration. The work considers a pair of transducers in a pitch-catch configuration on the surface of an isotropic metallic plate structure. The method's major drawbacks, as mentioned by the authors, include the inability to map the entirety of a sharp corner of a structure. The transducers are limited in their placement to the edges to avoid the risk of falling off the structure; thus, there is always an edge closer to the transducers than the corner point. Besides, gridlock situations occur also when the bounding box used to estimate the environment is inaccurate, resulting in inaccurate estimations, and it is impossible to distinguish between the complete mapping and gridlock without knowing the upper limit of obtainable coverage. In addition, transducers are manually positioned in the desired position, moving them instantaneously between

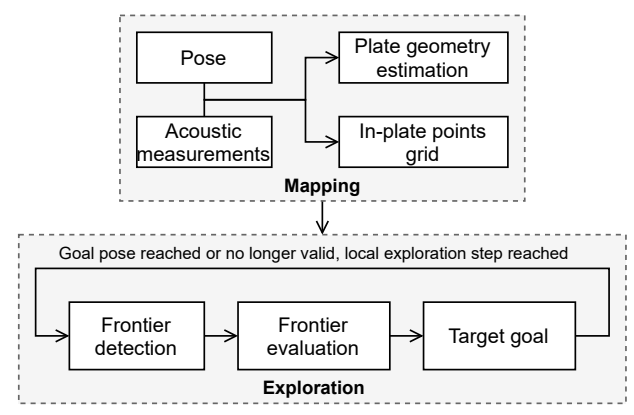

Fig. 2: Block diagram of the proposed method.

two locations. However, in a real-world scenario, a mobile robot moves continuously to the desired position, gathering more information about its surroundings, and the optimality of the chosen goal doesn't necessarily persist.

\section{METHOD}

A mobile robot equipped with a co-located transmitter/receiver pair of transducers and moving on a metal surface is considered. The emitter sends a pulse $s(t)$ to excite guided waves in the plate material at each scanning phase, and the receiver collects the acoustic response $z(t)$ containing the ultrasonic echoes. We restrict ourselves to mapping assuming a well-known position and frontier-based exploration is taken as the basic framework. We seek to find the optimum frontier points to take autonomous action to reconstruct the geometry of the plate in a fast manner, without getting too close to the plate's boundaries. Plate estimation and In-plate Points Grid update are kept running in the background, which continuously integrate robot pose and acoustic measurements. At each exploration step, the following steps are followed:

- Frontier points are extracted from the grid map.

- A utility function is used to evaluate the potential destinations.

- The candidate pose with the highest utility is selected as the next goal.

- The robot navigates to the target position.

The map is continuously updated as the robot moves toward the goal. If the goal is reached or is no longer valid (discussed in more details in section III.D.) or if the local exploration step is attained, the exploration process is run again. The frontier exploration is considered complete when there are no more frontier points to evaluate regarding their distance to the edge. A graphical overview of the proposed approach is presented in Fig. 2.

\section{A. Plate geometry estimation}

As in [2], echo detection and plate geometry reconstruction are based on a propagation model which is used to construct $\hat{z}(r, t)$, the expected signal that would be received if the incident wave is reflected at a distance $r$ to the transducers. Given measurement $z_{i}(t)$, the correlation signal:

$$
z_{i}^{\prime}(r)=\frac{\left\langle z_{i}(t), \hat{z}(r, t)\right\rangle}{\sqrt{\left\langle z_{i}(t), z_{i}(t)\right\rangle\langle\hat{z}(r, t), \hat{z}(r, t)\rangle}}
$$


is computed and its envelope:

$$
e_{i}(r)=\left|z_{i}^{\prime}(r)+j \mathcal{H}\left(z_{i}^{\prime}\right)(r)\right|
$$

is retrieved, as the most likely first-order reflections can be identified by its local maxima. Next, plate boundaries are represented by 2D lines with parameters $(r, \theta)$ which define the line equation with:

$$
x \cdot \cos \theta+y \cdot \sin \theta-r=0 .
$$

These boundaries are subsequently detected by constructing first the beamforming map given measurements $z_{1 . . T}$ obtained all along the robot trajectory $\mathbf{x}_{1 . . T}$ :

$$
\mathcal{L}_{T}(r, \theta)=\sum_{i=1}^{T} e_{i}\left(\left|x_{i} \cdot \cos \theta+y_{i} \cdot \sin \theta-r\right|\right)
$$

where $\mathbf{x}_{i}=\left(x_{i}, y_{i}\right)$ is the robot position during time-step $i$. Next, the optimization problem:

$$
\hat{\mathbf{M}}=\arg \max _{\mathbf{M}} \mathcal{L}_{T}(\mathbf{M})=\arg \max _{\mathbf{M}} \sum_{l=1}^{4} \mathcal{L}_{T}\left(r_{l}, \theta_{l}\right)
$$

is solved with the method described in [2] to identify the map $\mathbf{M}=\left\{r_{l}, \theta_{l}\right\}_{l=1 \ldots 4}$, where the four lines are restricted to define a rectangle altogether.

\section{B. In-plate Points Grid}

Our construction of the grid is equivalent to the occupancy grid mapping framework [11]. Let the In-Plate grid $\mathbf{G}$ be decomposed into $n \times m$ evenly-spaced grid cells where the i-th grid cell $c_{i}$ is assigned a static binary variable $o\left(c_{i}\right)$ for $i \in\{1, \ldots, n \times m\}$ that is defined as $o\left(c_{i}\right)=0$ when $c_{i}$ is inside the plate and $o\left(c_{i}\right)=1$ otherwise.

The grid's construction relies on detecting the closest edge to the sensor to identify which areas are inside the plate. And, we use the map $\hat{\mathbf{M}}$ to estimate the direction of arrival of the echo within a Bayesian framework to determine which areas are outside the plate. The beamforming map $\mathcal{L}_{T}(r, \theta)$ also uses the grid's information to filter the lines with parameters $(r, \theta)$ such as a line $l=\left(r_{l}, \theta_{l}\right)$ is filtered if it contains a point which is inside the plate. Overall, as the coverage of explored area increases, more lines of the beamforming map are filtered, indirectly increasing the plate's geometry estimation recovery speed. Furthermore, due to page limit constraints, this mapping approach is not detailed here.

\section{Frontier generation and evaluation}

After each In-plate Points Grid update, each grid cell has a state probability. We use a thresholding method to assign discrete states obtaining then a deterministic world model [12]. We choose a threshold $\delta$ for which a grid cell is labeled as in-plate for a state probability lower than $\delta$ and unknown otherwise. Frontier points are then generated based on an edge detection technique borrowed from computer vision [13].

The frontier points evaluation function is the basis for frontier points selection. We evaluate the frontier points from

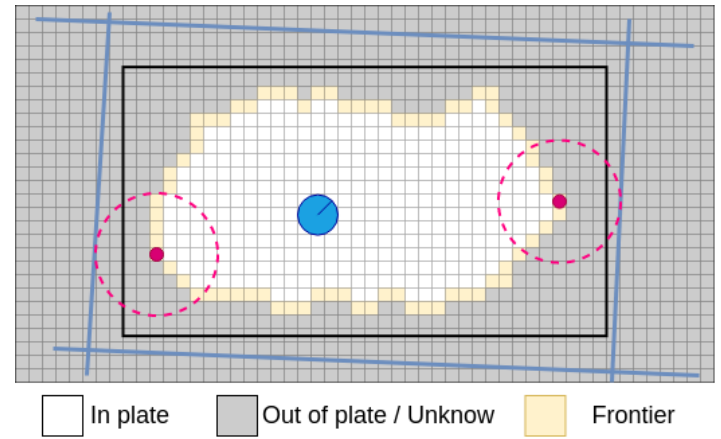

Fig. 3: Graphic representation illustrating an example of the expected area to explore (bounded by the dashed pink circle) for two frontier points (pink dots) given the plate geometry estimation (blue lines). The black rectangle illustrates the true outline of the plate. For simplicity, gray cells are used to illustrate both out of the plate and unknown cells.

the following two factors: information gain at the frontier point and the Euclidean distance to the robot's pose.

The information gain is defined as the area of an unknown region expected to be explored for a given frontier point $p_{k}$ and quantified using Shannon entropy:

$$
I\left(p_{k}, \hat{\mathbf{M}}\right)=\sum_{c \in V_{k}} e(c)
$$

where $V_{k}$ is the set of grid cells contained in the expected area of measurement defined by a circle centered in the frontier point $p_{k}$ with a radius equivalent to the distance to the closest edge given the plate's estimation $\hat{\mathbf{M}}$ (Fig. 3), and $e(c)$ refers to the entropy of the probability distribution $\hat{o}_{t}(c)$ such as:

$$
e(c)=-\hat{o}_{t}(c) \log \left(\hat{o}_{t}(c)\right)-\left(1-\hat{o}_{t}(c)\right) \log \left(1-\hat{o}_{t}(c)\right)
$$

We note $\hat{o}_{t}\left(c_{i}\right)=p\left(o\left(c_{i}\right) / x_{1: t}, z_{1: t}\right)$ the in-plate probability of grid $c_{i}$ where $z_{1: t}$ the set of all measurements up to time $\mathrm{t}$, and $x_{1: t}$ is the path of the robot defined through the sequence of all poses. We evaluate both unknown regions and regions where the map is still uncertain by taking into account the entropy of both observed and unobserved grid cells.

The second factor is the Euclidean distance $d\left(p_{k}\right)$ from the sensor's pose to the frontier point $p_{k}$. Each factor of the utility function is subjected to a min-max normalization to map its values to a range between 0 and 1 :

$$
\hat{f}_{k}=\frac{f_{k}-\min (\mathbf{f})}{\max (\mathbf{f})-\min (\mathbf{f})}
$$

where $f_{k}$ is the factor associated to the frontier point $p_{k}$ and $f$ the set containing the factor associated to all frontier points. Based on both factors, the utility function is then defined as:

$$
U\left(p_{k}, \hat{\mathbf{M}}\right)=\alpha\left(1-\hat{d}\left(p_{k}\right)\right)+(1-\alpha) \hat{I}\left(p_{k}, \hat{\mathbf{M}}\right)
$$

where $\hat{d}\left(p_{k}\right)$ and $\hat{I}\left(p_{k}, \hat{\mathbf{M}}\right)$ are the normalized factors and $\alpha$ is a weight parameter that varies between 0 and 1 to adjust the importance of each factor. Given $N$ nominee points, the next goal is the point with the highest utility evaluation as:

$$
p_{\text {opt }}=\underset{p_{k}}{\operatorname{argmax}}\left\{U\left(p_{k}, \hat{\mathbf{M}}\right), k \in[1, N]\right\}
$$


D. Validity of frontier points and exploration stopping condition

We create another grid layer naming it Radius Grid R (Fig. 4b) with the same dimensions as the In-plate Points Grid G. $\mathbf{R}$ is updated simultaneously as $\mathbf{G}$ using the robot pose $\mathbf{x}_{t}$ and the echo retrieved from the measurement $z_{t}$. The goal of this grid is to define the validity of frontier points in terms of their estimated distance from the true edge to avoid crossing any plate boundaries. For each grid cell, $g_{i}$ is assigned the minimum measured echo that passed by this cell. The validity of a frontier point $p_{i}$ is defined by its associated Radius Grid value; if this value is less than a defined threshold $\rho, p_{i}$ is then labeled as an invalid candidate point. This method, which uses the first echo, is a heuristic that gives information if the sensor is too close to a real edge. It also allows us to set a stopping condition for the exploration process: there are no more valid candidate points. Fig. 4 illustrates the In-plate Points Grid as well as the parallel Radius Grid layer defining valid and invalid frontier points for $\rho=0.2 \mathrm{~m}$.

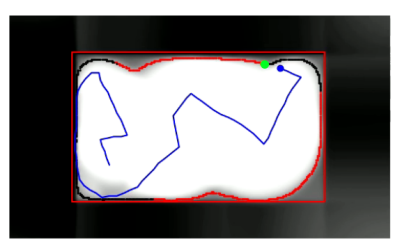

(a)

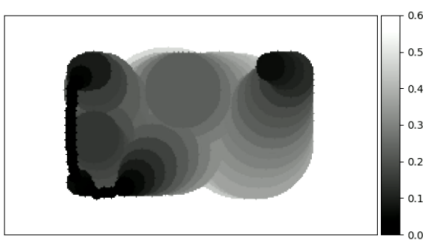

(b)
Fig. 4: (a) The red rectangle represents the estimated map in simulation of a $1.7 \times 1 \mathrm{~m}^{2}$ plate. The sensor position and path are represented by the blue dot and blue polyline. The red (resp. black) dots illustrate valid (resp. invalid) frontier points; (b) The associated Radius Grid $\mathbf{R}$.

\section{E. Repetitive Re-checking and the exploration algorithm}

The map is updated continuously during navigation to the selected location. As a result, some new frontier points will be generated, some old frontiers will no longer be valid, and the selected point may no longer be the optimal target. It is, therefore, unnecessary to continue traveling to the chosen location [6], [14]. We address this problem by defining a local exploration path step size $s_{\text {exp }}$. Each time when the traveled distance of the robot reaches the step size, the next optimal target is selected using the exploration strategy. Algorithm 1 summarizes the overall implementation of our exploration strategy:

\section{EXPERIMENTAL EVALUATION}

To evaluate the feasibility and viability of our approach, we present several tests conducted in simulation and with a real robot. We measure, as exploration time progresses, the distance traveled by the robot and the percentage of covered area (as done, e.g., in [14]), namely the percentage of the area of cells labeled as inside the plate relative to the real area of the plate. For both the simulated and real-world experiments, we work on a flat rectangular metallic plate with dimensions $1700 \times 1000 \times 6 \mathrm{~mm}^{3}$ and we use $\alpha=0.7, \delta=0.3, s_{\text {exp }}=$ $20 \mathrm{~cm}, \rho=15 \mathrm{~cm}$ and the grid size $=1 \times 1 \mathrm{~cm}^{2}$.

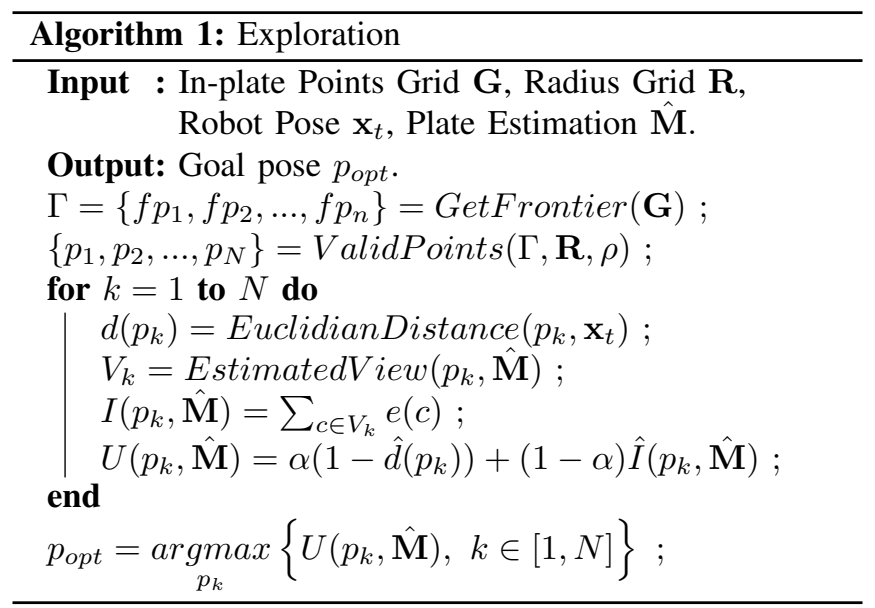

\section{A. Echo detection}

First, we illustrate the echo-detection principle. The emitted signal is a 2-cycle burst at $100 \mathrm{kHz}$. We show, in Fig. 5, the measured acoustic signal $z_{i}(t)$ for a sensor position (28, 50) centimeters relative to the plate's corner. Fig. 5.b shows the resulting correlation signal $z_{i}^{\prime}(r)$ computed using eq.(1) and its envelope $e_{i}(r)$ calculated with eq.(2) as explained in [2].

The method relies on the detection of the first peak (local maxima) in the correlation using peak properties. In this case, prominence is used as the main property. The prominence of a peak is defined as the shortest drop in altitude required to reach any higher terrain from the summit and is used, here, to distinguish the echo from noisy peaks, because the higher the prominence, the more important the peak is.

To automate the process of peak detection, first, we retrieve all the peaks of the correlation and calculate the prominence of each peak. Next, we calculate the $k^{\text {th }}$ percentile $p^{k}$ of these values. Then, we recalculate the peaks with a required prominence higher than the percentile value found in the first step.

Fig. 5 illustrates the result of the method for a prominence value $p^{k}=85 \%$. The red line presents the peak detected at $29.3 \mathrm{~cm}$ and the green line presents the expected echo given the ground truth pose $(x, y)$ which is defined by $\min (x, y, w-x, h-y)$ where $(w, h)$ are the width and height of the plate. The error between the ground truth and peak detected in Fig. 5 is $1.4 \mathrm{~cm}$.

\section{B. Simulation}

Simulations are performed in a Python environment to evaluate the proposed method. We simulate the two colocated transducers as a particle with a position referring to the central position between the two. The signals are simulated using the measurement model based on the propagation model as explained in [2] and correlation is retrieved as shown in Fig. 5.

In Fig. 6, we show the In-plate Points Grid and the exploration path of the particle. We also represent the estimated geometry, the valid and invalid frontier points, and the selected candidate point based on the utility function 
a)
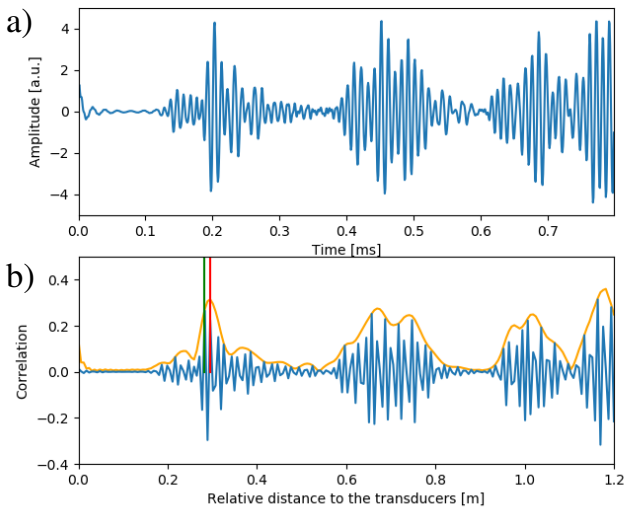

Fig. 5: Illustration of the echo detection principle for a real signal based on correlation with the propagation model in a $1700 \times 1000 \times 6 \mathrm{~mm}^{3}$ metallic plate. a) the acoustic measurement. b) representation of the correlation signal (blue) and its envelope (orange), the retrieved echo (red line) and ground true echo (green line) based on exterior localization.

defined. In step 1, the estimated plate is incorrectly estimated as expected since only one measurement was integrated. Because all of the points are within the same distance of the sensor position, only the expected area to explore is used to differentiate the utility of the points. The next goal is then randomly chosen from one of the four points pointing to the edges. As in step 41, the three closest edges and the orientation are well estimated. The sensor moved closer to the left part of the plate until no valid candidate points remained; consequently, the sensor went to the center of the plate to acquire the maximum area to explore. In step 83, the shape has been approximately fully recovered and $83 \%$ of the plate has been covered with the interior points grid. Leading to the final step 125, the sensor followed the remaining frontier points until there were no valid frontier points left, indicating the end of the frontier exploration process.

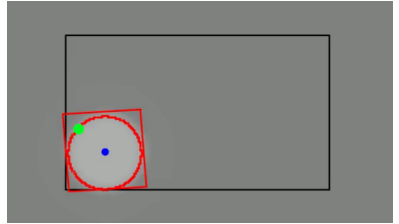

Step 1

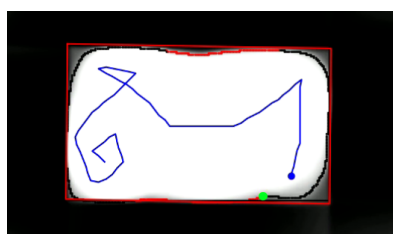

Step 83

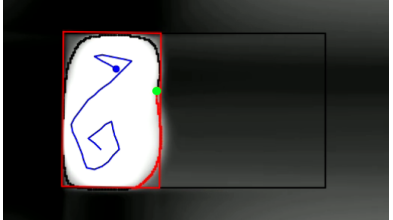

Step 41

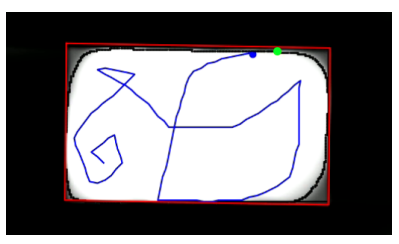

Step 125
Fig. 6: Interior points grid and path generated by the exploration algorithm The estimated plate is the red rectangle. The true outline of the plate and the true sensor positions correspond to the black rectangle and blue particle respectively. The valid (resp. invalid) frontier points correspond to the red (resp. black) dots. The green particle refers to the selected candidate point.

The following methods are compared to our approach: the classic frontier method (closest frontier point) [3], picking random points from frontier points, and our method using the true plate's geometry instead of the estimated map. In the case of closest and random frontier point selection, the sensor moves until it reaches the selected point before moving on to the next valid location. To have a fair comparison, we present results up to $83 \%$ of mean coverage, because some runs end without reaching full coverage. We show in Fig. 7 the average coverage increase calculated over 50 runs for each algorithm. The same starting position is used for each run corresponding to $20 \mathrm{~cm}$ to the plate's corner. We also represent the $10 \%$ and $90 \%$ quantiles to assess the repeatability of each approach.

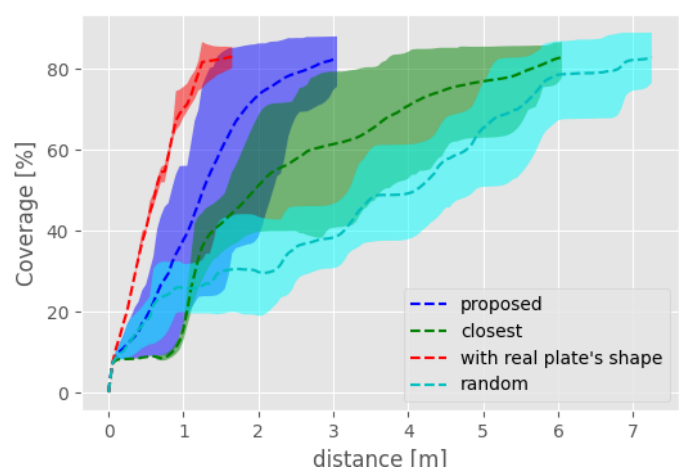

Fig. 7: Exploration results for the four exploration approaches in terms of coverage increase as a function of the traveled distance.

The method using the true plate's geometry, shown in red, produces an approximate deterministic result with a low standard deviation up to $63 \%$ of coverage, demonstrating the benefit of having a known map. The curve rises quickly, providing on average $70 \%$ coverage after $1 \mathrm{~m}$ of displacement. The closest selection method grows in a roughly linear fashion with an average coverage rate of $0.15 \% / \mathrm{cm}$ and presents a behavior approximately equal to the random approach with a higher standard deviation.

On the other hand, though a random approach will eventually produce a map, random choices may not provide an efficient sequence for mapping an environment. As expected, the approach underperforms when compared to other algorithms.

Our method, on average, outperforms random and closest frontier exploration as made evident by the mean result curves of Fig 7 as it takes into account information gain and navigation cost.

\section{Real-world experiment}

The origin of the xy-coordinate system is at the bottom left corner of the plate as shown in Fig. 1.b. As presented in Fig.1a, we use two co-located transducers fixed on a spring mechanism mounted on a TurtleBot to maintain constant contact with the surface. A layer of water is added to the plate's surface as a coupling medium for the transducers used in the acoustic measurements. Considering the projection on the plate of both the rotation center of the robot and the middle point between the two transducers, the distance between these two points is $32 \mathrm{~cm}$. We use two-tone bursts of a sinusoidal wave at $100 \mathrm{kHz}$ as the excitation and a sampling frequency of 1,25 MHz. Moreover, the direct incident signal is smoothly removed from the data as it does not correspond 


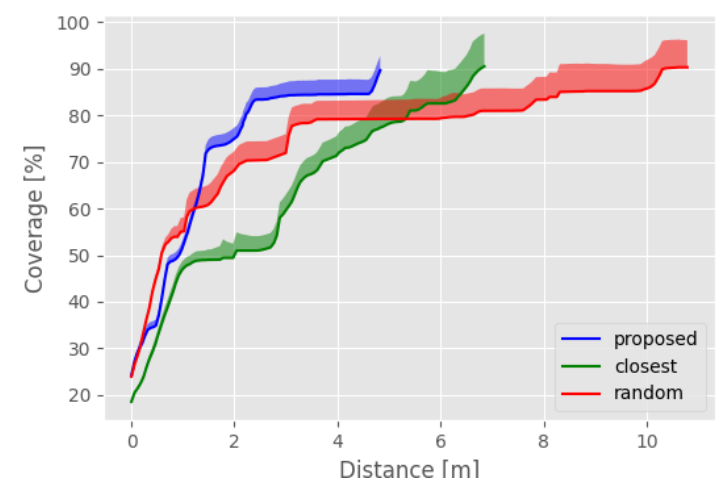

Fig. 8: Comparing the approaches in real-world experiment.

to a reflection on an edge. This filtering limits the distance at which the signal containing the information about the closest edge can be detected to about $15 \mathrm{~cm}$ limiting then the sensor's positioning from the edge. For values less than that, the first echo is misdetected, resulting in an In-plate Points Grid that crosses the real plate's boundaries.

Throughout all the experiments, we use a camera with AR tags tracking [15] as an external localization system as shown in Fig. 1a. Also, to evaluate the system independently of the quality of the controller, while the robot has a strong tendency to slide, the movement between waypoints is implemented with the joystick.

The two methods, namely closest and random frontier selection, are compared to our approach, as done in the simulation. As it may be seen in Fig. 1.a, the In-plate Points Grid exceeds the plate's real geometry mainly because of a misdetected closest edge. Several factors could cause the echo detection error, including changes in the propagation model due to the use of water as a couplant between the transducers and the plate, not enough water between the transducer and the plate, and human errors while positioning the ar_tags to locate the robot on the plate. The mapping method is sensitive to large misdetected echoes. In this case, lines representing the true edges could be filtered. The robot then risks exceeding the edges resulting in an overall incorrect geometry estimation.

We run each algorithm five times each with the same starting position corresponding to $(25 \mathrm{~cm}, 45 \mathrm{~cm})$. To have a fair comparison and similar to what we did in the simulation, we present results up to $91 \%$ of the mean coverage. Fig. 8 illustrates the mean coverage value. The upper bound is the full coverage computed during the exploration process, and the lower bound is the full coverage minus the error coverage (i.e. covered area outside the plate).

In the proposed method, the coverage percentage increases fast, reaching $83 \%$ with a displacement of $2.38 \mathrm{~m}$. We remark that the random approach outperforms our method at the beginning but plateaus around $80 \%$ coverage. The main reason is that random procedure may choose distant points, resulting in rapid coverage increase but fails to obtain all details at the end. The proposed exploration algorithm outperforms the two other methods balancing between traveling cost and information gain

\section{CONCLUSIONS}

In this paper, we present a novel application and integration of UGWs with the frontier exploration strategy, that takes into account the estimated geometry of the plate, balancing the information gain and the travel cost. Both in simulation and real-world experiments carried out in a laboratory environment, the proposed approach outperforms random and closest frontier selection in terms of coverage increase rate. The next step will be to integrate the exploration method with a SLAM framework to account for the state estimation error. Besides, the automated first echo detection can be further investigated to be more robust given that more complex and noisy signals are expected on a large metal structure and in an outdoor environment. Finally, robot dynamics can be considered when selecting frontier points to minimize velocity changes and maintain a consistently high speed for fast reconstruction and exploration of the plate.

\section{REFERENCES}

[1] CORDIS, "Bugwright2, autonomous robotic inspection and maintenance on ship hulls and storage tanks, description of the innovative action," 2019

[2] O. L. Ouabi, P. Pomarede, M. Geist, N. F. Declercq, and C. Pradalier, "A fastslam approach integrating beamforming maps for ultrasoundbased robotic inspection of metal structures," IEEE Robotics and Automation Letters, pp. 1-1, 2021.

[3] B. Yamauchi, "A frontier-based approach for autonomous exploration," in Proceedings 1997 IEEE International Symposium on Computational Intelligence in Robotics and Automation CIRA'97. 'Towards New Computational Principles for Robotics and Automation', 1997, pp. 146-151.

[4] M. Juliá, A. Gil, and Ó. Reinoso, "A comparison of path planning strategies for autonomous exploration and mapping of unknown environments," Autonomous Robots, vol. 33, pp. 427-444, 2012.

[5] A. Mobarhani, S. Nazari, A. H. Tamjidi, and H. D. Taghirad, "Histogram based frontier exploration," in 2011 IEEE/RSJ International Conference on Intelligent Robots and Systems, 2011, pp. 1128-1133.

[6] B. Fang, J. Ding, and Z. Wang, "Autonomous robotic exploration based on frontier point optimization and multistep path planning," IEEE Access, vol. 7, pp. 46 104-46113, 2019.

[7] H. H. González-Baños and J.-C. Latombe, "Navigation strategies for exploring indoor environments," The International Journal of Robotics Research, vol. 21, no. 10-11, pp. 829-848, 2002.

[8] F. Amigoni and A. Gallo, "A multi-objective exploration strategy for mobile robots," in Proceedings of the 2005 IEEE International Conference on Robotics and Automation, 2005, pp. 3850-3855

[9] F. Bourgault, A. Makarenko, S. Williams, B. Grocholsky, and H. Durrant-Whyte, "Information based adaptive robotic exploration," in IEEE/RSJ International Conference on Intelligent Robots and Systems, vol. 1, 2002, pp. 540-545 vol.1.

[10] A. Miranda, J. V. Hook, and C. Schaal, "Lamb wave-based mapping of plate structures via frontier exploration," Ultrasonics, vol. 110, p. 106282, 2021.

[11] S. Thrun, W. Burgard, and D. Fox, Probabilistic Robotics (Intelligent Robotics and Autonomous Agents). The MIT Press, 2005.

[12] H. Moravec and A. Elfes, "High resolution maps from wide angle sonar," in Proceedings. 1985 IEEE International Conference on Robotics and Automation, vol. 2, 1985, pp. 116-121.

[13] L. Roberts, Machine Perception of Three-Dimensional Solids, 011963.

[14] D. Holz, N. Basilico, F. Amigoni, and S. Behnke, "A comparative evaluation of exploration strategies and heuristics to improve them," in ECMR, 2011.

[15] S. Niekum, "Ros package: ar_track_alvar." [Online]. Available: http://wiki.ros.org/ar_track_alvar 